\title{
Structure-Based Approach for the Study of Thyroid Hormone \\ Receptor Binding Affinity and Subtype Selectivity
}

Fang-Fang Wang ${ }^{\text {a }}$, Wei Yang ${ }^{\mathrm{b}}$, Yong-Hui Shi ${ }^{\mathrm{a}}$, Xiang-Rong Chenga, Guow-Wei Le ${ }^{\text {a** }}$

${ }^{a}$ The State Key Laboratory of Food Science and Technology, School of Food Science and

Technology, Jiangnan University, Wuxi, 214122, China.

${ }^{\mathrm{b}}$ Department of Biochemistry and Molecular Biology, Faculty of Medicine, Monash University, Melbourne, VIC 3800, Australia.

* Corresponding author: Tel.: +86-510-8591-7789; Fax: + 86-510-8591-7789.

E-mail: lgw@jiangnan.edu.cn

Table S1. Molecular structures of Indane Derivatives and their TR $\beta$ binding affinity values $\left(\mathrm{pK}_{\mathrm{i}}\right)$.

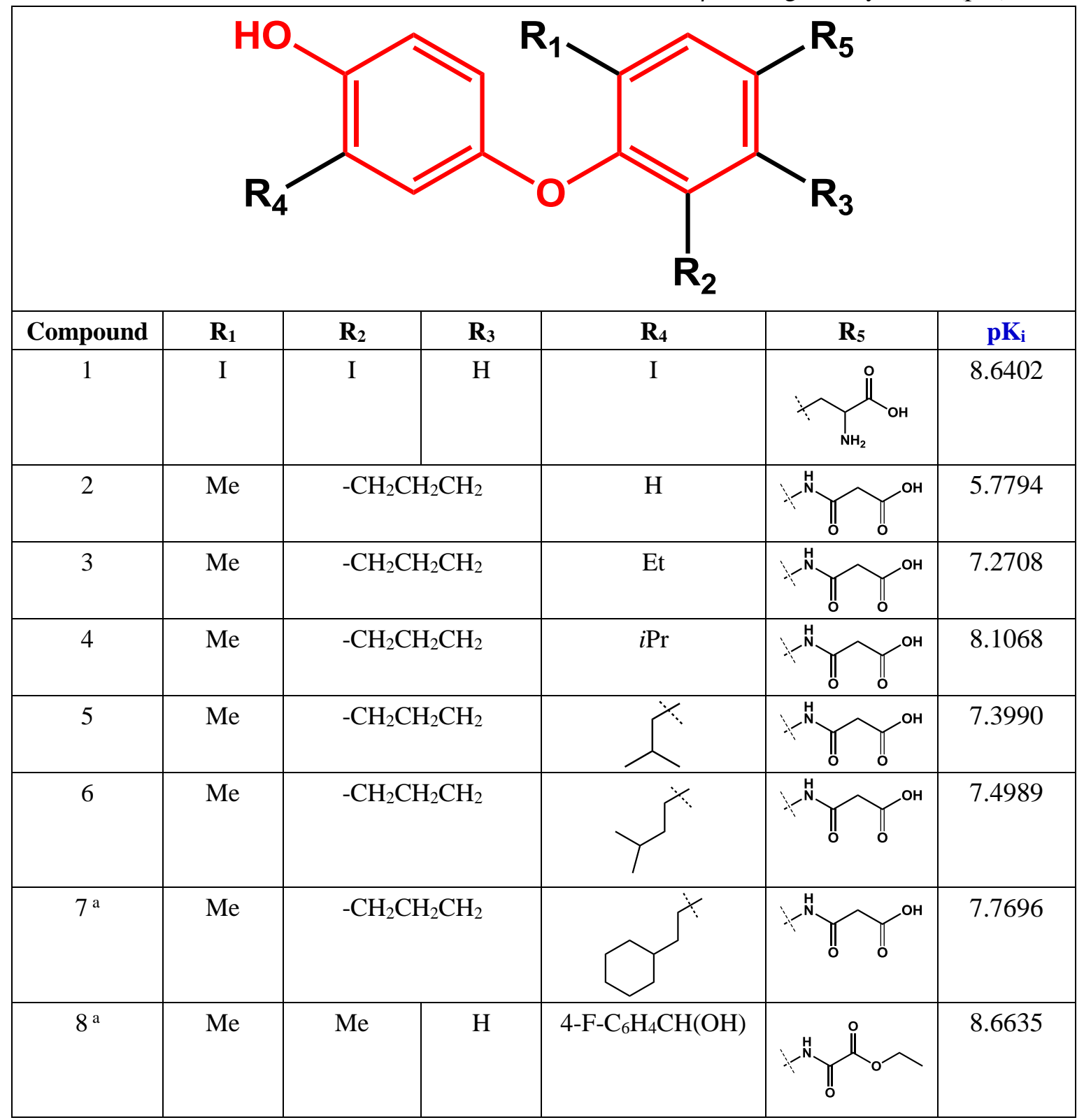




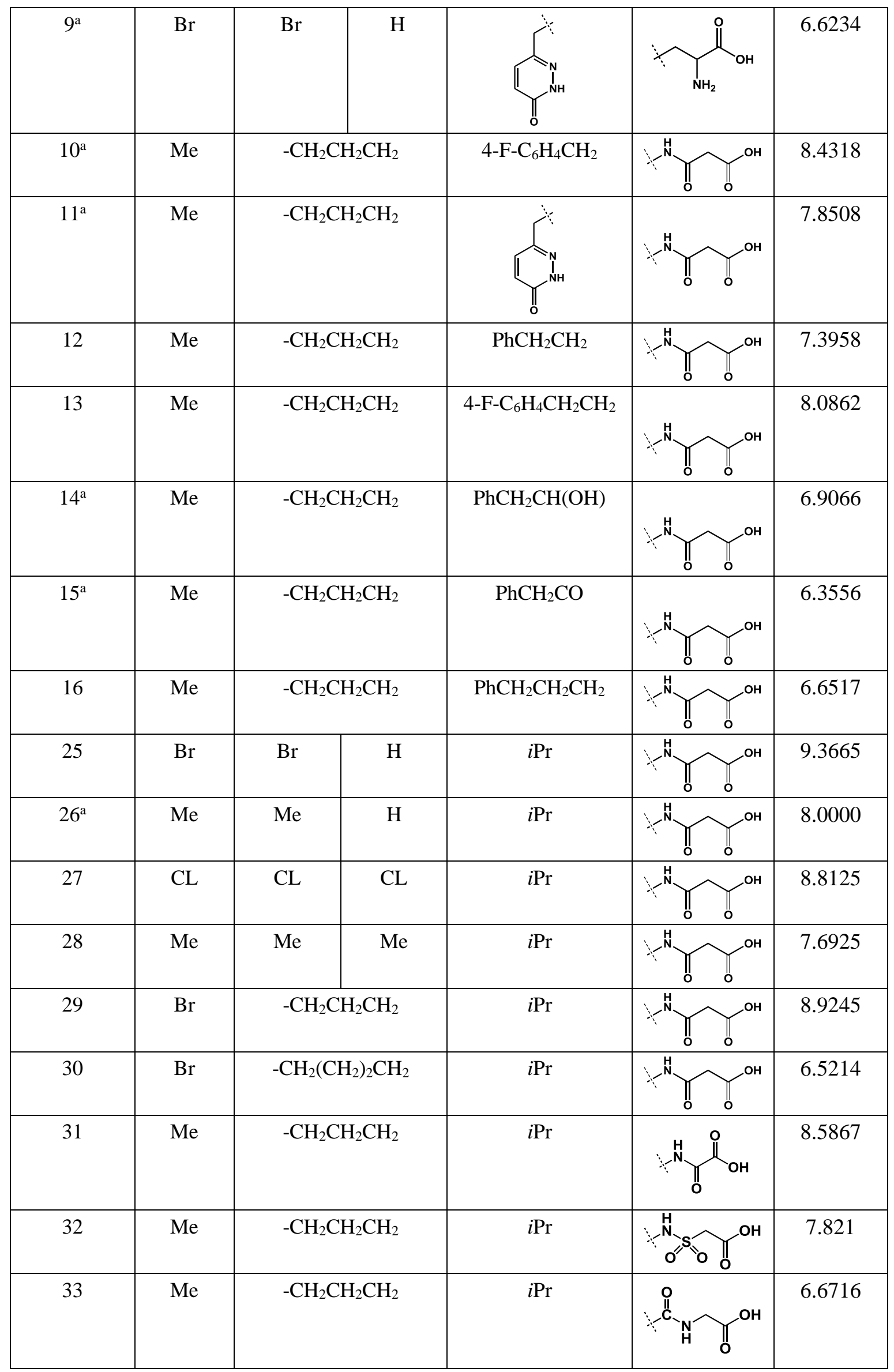




\begin{tabular}{|c|c|c|c|c|}
\hline \multicolumn{1}{|c|}{} \\
\hline Compound & 2 & 3 & 4 & PKinM) \\
\hline 17 & $\mathrm{~F}$ & $\mathrm{H}$ & $\mathrm{F}$ & 7.9208 \\
\hline 18 & $\mathrm{H}$ & $\mathrm{H}$ & $\mathrm{H}$ & 8.2218 \\
\hline $19^{\mathrm{a}}$ & $\mathrm{CL}$ & $\mathrm{CL}$ & $\mathrm{H}$ & 7.5229 \\
\hline 20 & $\mathrm{H}$ & $\mathrm{H}$ & $\mathrm{CL}$ & 7.3872 \\
\hline 21 & $\mathrm{H}$ & $\mathrm{H}$ & $\mathrm{H}$ & 7.2716 \\
\hline 22 & $\mathrm{OH}$ & $\mathrm{OH}$ & $\mathrm{H}$ & 7.0899 \\
\hline 23 & $\mathrm{H}$ & $\mathrm{H}$ & $\mathrm{OH}$ & 7.2396 \\
\hline 24 & $\mathrm{H}$ &
\end{tabular}

${ }^{\mathrm{a}}$ represents the test set.

Table S2. Summary of QSAR results based on superimposition I .

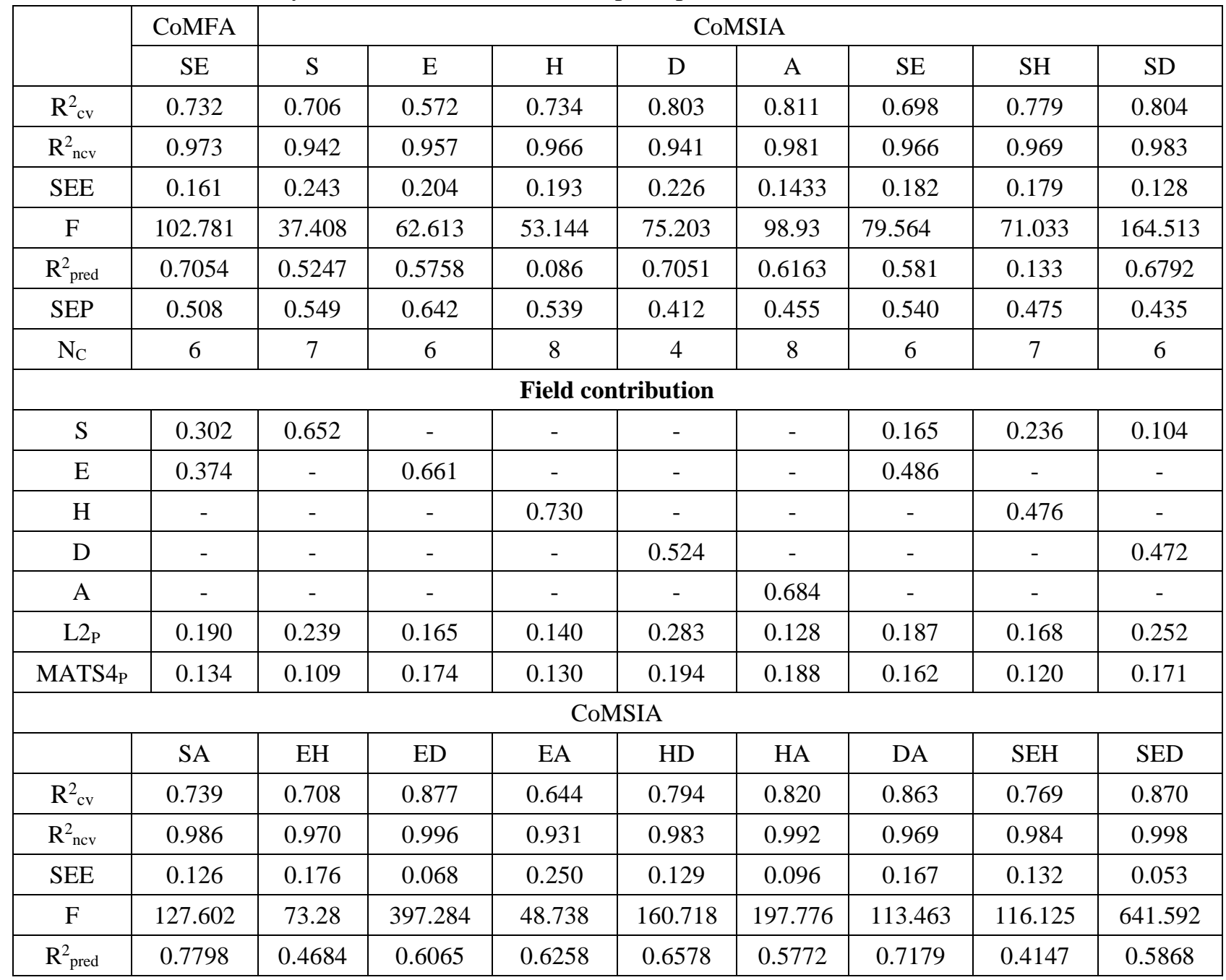




\begin{tabular}{|c|c|c|c|c|c|c|c|c|c|}
\hline SEP & 0.534 & 0.547 & 0.379 & 0.569 & 0.445 & 0.459 & 0.353 & 0.502 & 0.390 \\
\hline $\mathrm{N}_{\mathrm{C}}$ & 8 & 7 & 9 & 5 & 6 & 9 & 5 & 8 & 9 \\
\hline \multicolumn{10}{|c|}{ Field contribution } \\
\hline$S$ & 0.208 & - & - & - & - & - & - & 0.129 & 0.070 \\
\hline $\mathrm{E}$ & - & 0.406 & 0.259 & 0.362 & - & - & - & 0.346 & 0.236 \\
\hline $\mathrm{H}$ & - & 0.271 & - & - & 0.193 & 0.336 & - & 0.230 & - \\
\hline $\mathrm{D}$ & - & - & 0.347 & - & 0.412 & - & 0.380 & - & 0.305 \\
\hline A & 0.427 & - & - & 0.264 & - & 0.330 & 0.181 & - & - \\
\hline $\mathrm{L} 2_{\mathrm{P}}$ & 0.191 & 0.167 & 0.229 & 0.175 & 0.227 & 0.163 & 0.245 & 0.165 & 0.228 \\
\hline MATS4 $_{\mathrm{P}}$ & 0.173 & 0.156 & 0.166 & 0.199 & 0.168 & 0.171 & 0.195 & 0.129 & 0.160 \\
\hline \multicolumn{10}{|c|}{ CoMSIA } \\
\hline & SEA & SHD & SHA & SDA & EHD & EHA & EDA & HDA & SEHD \\
\hline $\mathrm{R}_{\mathrm{cv}}^{2}$ & 0.718 & 0.810 & 0.802 & 0.848 & 0.858 & 0.753 & 0.890 & 0.846 & 0.867 \\
\hline $\mathrm{R}_{\mathrm{ncv}}^{2}$ & 0.989 & 0.972 & 0.991 & 0.971 & 0.995 & 0.986 & 0.996 & 0.982 & 0.999 \\
\hline SEE & 0.107 & 0.160 & 0.100 & 0.163 & 0.075 & 0.124 & 0.066 & 0.133 & 0.040 \\
\hline $\mathrm{F}$ & 176.463 & 123.864 & 180.022 & 119.555 & 364.58 & 130.579 & 411.521 & 152.426 & 1040.82 \\
\hline $\mathrm{R}_{\text {pred }}^{2}$ & 0.6966 & 0.6862 & 0.601 & 0.7205 & 0.5908 & 0.5851 & 0.6356 & 0.7115 & 0.5149 \\
\hline SEP & 0.555 & 0.428 & 0.481 & 0.372 & 0.394 & 0.520 & 0.359 & 0.385 & 0.410 \\
\hline $\mathrm{N}_{\mathrm{C}}$ & 8 & 5 & 9 & 5 & 8 & 8 & 9 & 6 & 10 \\
\hline \multicolumn{10}{|c|}{ Field contribution } \\
\hline $\mathrm{S}$ & 0.134 & 0.078 & 0.145 & 0.074 & - & - & - & - & 0.065 \\
\hline E & 0.334 & - & - & - & 0.212 & 0.303 & 0.200 & - & 0.200 \\
\hline $\mathrm{H}$ & - & 0.162 & 0.261 & - & 0.119 & 0.196 & - & 0.144 & 0.113 \\
\hline $\mathrm{D}$ & - & 0.361 & - & 0.338 & 0.290 & - & 0.281 & 0.313 & 0.256 \\
\hline $\mathrm{A}$ & 0.220 & - & 0.266 & 0.159 & - & 0.191 & 0.124 & 0.153 & - \\
\hline L2P & 0.174 & 0.231 & 0.169 & 0.242 & 0.219 & 0.165 & 0.221 & 0.213 & 0.213 \\
\hline MATS4$_{P}$ & 0.138 & 0.168 & 0.159 & 0.187 & 0.161 & 0.146 & 0.175 & 0.177 & 0.153 \\
\hline \multicolumn{10}{|c|}{ CoMSIA } \\
\hline & \multicolumn{2}{|l|}{ SEHA } & SEDA & \multicolumn{2}{|c|}{ SHDA } & \multicolumn{2}{|l|}{ EHDA } & \multicolumn{2}{|c|}{ SEHDA } \\
\hline $\mathrm{R}_{\mathrm{cv}}^{2}$ & \multicolumn{2}{|l|}{0.783} & 0.881 & \multicolumn{2}{|c|}{0.853} & \multicolumn{2}{|l|}{0.873} & \multicolumn{2}{|c|}{0.875} \\
\hline $\mathrm{R}_{\text {ncv }}^{2}$ & \multicolumn{2}{|l|}{0.993} & 0.997 & \multicolumn{2}{|c|}{0.982} & \multicolumn{2}{|l|}{0.995} & \multicolumn{2}{|c|}{0.995} \\
\hline SEE & \multicolumn{2}{|l|}{0.088} & 0.059 & \multicolumn{2}{|c|}{0.132} & \multicolumn{2}{|l|}{0.077} & \multicolumn{2}{|c|}{0.075} \\
\hline $\mathrm{F}$ & \multicolumn{2}{|l|}{231.901} & 529.767 & \multicolumn{2}{|c|}{154.502} & 341.78 & & 364.12 & \\
\hline $\mathrm{R}_{\text {pred }}^{2}$ & 0.5868 & & 0.6301 & 0.7 & & 0.6286 & & 0.6336 & \\
\hline SEP & 0.504 & & 0.373 & 0.3 & & 0.372 & & 0.370 & \\
\hline $\mathrm{N}_{\mathrm{C}}$ & 9 & & 9 & 6 & & 8 & & 8 & \\
\hline & & & & Field co & ribution & & & & \\
\hline$S$ & 0.106 & & 0.059 & 0.0 & & - & & 0.051 & \\
\hline E & 0.252 & & 0.181 & - & & 0.171 & & 0.158 & \\
\hline $\mathrm{H}$ & 0.174 & & - & 0.1 & & 0.099 & & 0.093 & \\
\hline $\mathrm{D}$ & - & & 0.251 & 0.2 & & 0.245 & & 0.225 & \\
\hline $\mathrm{A}$ & 0.164 & & 0.115 & 0.1 & & 0.109 & & 0.100 & \\
\hline $\mathrm{L} 2 \mathrm{P}$ & 0.177 & & 0.223 & 0.2 & & 0.210 & & 0.209 & \\
\hline
\end{tabular}




\begin{tabular}{|l|l|l|l|l|l|}
\hline MATS4$_{\mathrm{P}}$ & 0.127 & 0.171 & 0.173 & 0.167 & 0.164 \\
\hline
\end{tabular}

Table S3. Summary of QSAR results based on superimposition II .

\begin{tabular}{|c|c|c|c|c|c|c|c|c|c|}
\hline & & \multicolumn{8}{|c|}{ CoMSIA } \\
\hline & SE & $\mathrm{S}$ & $\mathrm{E}$ & $\mathrm{H}$ & $\mathrm{D}$ & A & $\mathrm{SE}$ & $\mathrm{SH}$ & SD \\
\hline $\mathrm{R}_{\mathrm{cV}}^{2}$ & 0.467 & 0.473 & 0.445 & 0.455 & 0.457 & 0.433 & 0.447 & 0.461 & 0.457 \\
\hline $\mathrm{R}_{\mathrm{ncv}}^{2}$ & 0.663 & 0.710 & 0.622 & 0.610 & 0.630 & 0.605 & 0.633 & 0.620 & 0.641 \\
\hline SEE & 0.513 & 0.487 & 0.543 & 0.552 & 0.537 & 0.555 & 0.535 & 0.544 & 0.529 \\
\hline $\mathrm{F}$ & 20.658 & 16.349 & 78.362 & 16.424 & 17.874 & 16.077 & 18.106 & 17.150 & 18.749 \\
\hline $\mathrm{R}_{\text {pred }}^{2}$ & 0.7262 & 0.6268 & 0.7192 & 0.7465 & 0.7583 & 0.7299 & 0.7165 & 0.7417 & 0.7521 \\
\hline SEP & 0.645 & 0.657 & 0.658 & 0.652 & 0.651 & 0.665 & 0.657 & 0.649 & 0.651 \\
\hline $\mathrm{N}_{\mathrm{C}}$ & 2 & 3 & 2 & 2 & 2 & 2 & 2 & 2 & 2 \\
\hline \multicolumn{10}{|c|}{ Field contribution } \\
\hline $\mathrm{S}$ & 0.113 & 0.433 & - & - & - & - & 0.045 & 0.044 & 0.044 \\
\hline $\mathrm{E}$ & 0.190 & - & 0.148 & - & - & - & 0.150 & - & - \\
\hline $\mathrm{H}$ & - & - & - & 0.083 & - & - & - & 0.085 & - \\
\hline $\mathrm{D}$ & - & - & - & - & 0.169 & - & - & - & 0.173 \\
\hline A & - & - & - & - & - & 0.068 & - & - & - \\
\hline GATS8e & 0.572 & 0.380 & 0.658 & 0.697 & 0.650 & 0.692 & 0.634 & 0.676 & 0.626 \\
\hline Mor29m & 0.125 & 0.187 & 0.194 & 0.220 & 0.181 & 0.240 & 0.171 & 0.194 & 0.157 \\
\hline \multicolumn{10}{|c|}{ CoMSIA } \\
\hline & SA & $\mathrm{EH}$ & ED & EA & HD & HA & DA & SEH & SED \\
\hline $\mathrm{R}_{\mathrm{cv}}^{2}$ & 0.438 & 0.439 & 0.433 & 0.425 & 0.445 & 0.431 & 0.429 & 0.440 & 0.433 \\
\hline $\mathrm{R}_{\mathrm{ncv}}^{2}$ & 0.615 & 0.638 & 0.612 & 0.601 & 0.646 & 0.620 & 0.607 & 0.648 & 0.615 \\
\hline SEE & 0.548 & 0.532 & 0.538 & 0.545 & 0.526 & 0.545 & 0.541 & 0.524 & 0.536 \\
\hline $\mathrm{F}$ & 16.785 & 18.484 & 34.723 & 33.153 & 19.132 & 17.131 & 33.934 & 19.371 & 35.075 \\
\hline $\mathrm{R}_{\text {pred }}^{2}$ & 0.7293 & 0.7290 & 0.6535 & 0.6348 & 0.7571 & 0.7405 & 0.6675 & 0.7230 & 0.6546 \\
\hline SEP & 0.662 & 0.662 & 0.650 & 0.655 & 0.658 & 0.666 & 0.652 & 0.661 & 0.650 \\
\hline $\mathrm{N}_{\mathrm{C}}$ & 2 & 2 & 1 & 1 & 2 & 2 & 1 & 2 & 1 \\
\hline \multicolumn{10}{|c|}{ Field contribution } \\
\hline $\mathrm{S}$ & 0.043 & - & - & - & - & - & - & 0.045 & 0.017 \\
\hline $\mathrm{E}$ & - & 0.149 & 0.078 & 0.082 & - & - & - & 0.147 & 0.077 \\
\hline $\mathrm{H}$ & - & 0.085 & - & - & 0.083 & 0.085 & - & 0.084 & - \\
\hline $\mathrm{D}$ & - & - & 0.085 & - & 0.170 & - & 0.088 & - & 0.084 \\
\hline $\mathrm{A}$ & 0.072 & - & - & 0.040 & - & 0.072 & 0.040 & - & - \\
\hline GATS8e & 0.677 & 0.614 & 0.540 & 0.566 & 0.607 & 0.658 & 0.562 & 0.586 & 0.531 \\
\hline Mor29m & 0.209 & 0.153 & 0.297 & 0.312 & 0.140 & 0.185 & 0.310 & 0.139 & 0.293 \\
\hline \multicolumn{10}{|c|}{ CoMSIA } \\
\hline & SEA & SHD & SHA & $\mathrm{SDA}$ & EHD & EHA & EDA & HDA & SEHD \\
\hline $\mathrm{R}_{\mathrm{cv}}^{2}$ & 0.425 & 0.444 & 0.432 & 0.429 & 0.430 & 0.422 & 0.427 & 0.426 & 0.430 \\
\hline $\mathrm{R}_{\mathrm{ncv}}^{2}$ & 0.604 & 0.657 & 0.631 & 0.609 & 0.613 & 0.603 & 0.614 & 0.608 & 0.615 \\
\hline SEE & 0.543 & 0.518 & 0.537 & 0.540 & 0.537 & 0.544 & 0.536 & 0.540 & 0.535 \\
\hline $\mathrm{F}$ & 33.519 & 20.087 & 17.964 & 34.299 & 34.836 & 33.357 & 34.998 & 34.100 & 35.167 \\
\hline
\end{tabular}




\begin{tabular}{|c|c|c|c|c|c|c|c|c|c|}
\hline $\mathrm{R}_{\text {pred }}^{2}$ & 0.6360 & 0.7478 & 0.7320 & 0.6687 & 0.6601 & 0.6421 & 0.6466 & 0.6744 & 0.6610 \\
\hline SEP & 0.655 & 0.659 & 0.666 & 0.652 & 0.652 & 0.656 & 0.653 & 0.654 & 0.652 \\
\hline $\mathrm{N}_{\mathrm{C}}$ & 1 & 2 & 2 & 1 & 1 & 1 & 1 & 1 & 1 \\
\hline \multicolumn{10}{|c|}{ Field contribution } \\
\hline$S$ & 0.017 & 0.045 & 0.045 & 0.017 & - & - & - & - & 0.016 \\
\hline $\mathrm{E}$ & 0.080 & - & - & - & 0.074 & 0.078 & 0.075 & - & 0.073 \\
\hline $\mathrm{H}$ & - & 0.082 & 0.086 & - & 0.046 & 0.048 & - & 0.048 & 0.045 \\
\hline D & - & 0.168 & - & 0.087 & 0.081 & - & 0.082 & 0.084 & 0.080 \\
\hline $\mathrm{A}$ & 0.039 & - & 0.073 & 0.039 & - & 0.038 & 0.037 & 0.038 & - \\
\hline GATS8e & 0.556 & 0.580 & 0.632 & 0.552 & 0.515 & 0.539 & 0.520 & 0.535 & 0.507 \\
\hline Mor29m & 0.307 & 0.126 & 0.163 & 0.304 & 0.284 & 0.297 & 0.287 & 0.295 & 0.279 \\
\hline \multicolumn{10}{|c|}{ CoMSIA } \\
\hline & \multicolumn{2}{|l|}{ SEHA } & SEDA & \multicolumn{2}{|c|}{ SHDA } & \multicolumn{2}{|c|}{ EHDA } & \multicolumn{2}{|c|}{ SEHDA } \\
\hline $\mathrm{R}_{\mathrm{cv}}^{2}$ & \multicolumn{2}{|l|}{0.422} & 0.427 & \multicolumn{2}{|c|}{0.426} & \multicolumn{2}{|c|}{0.424} & \multicolumn{2}{|c|}{0.424} \\
\hline $\mathrm{R}_{\mathrm{ncv}}^{2}$ & \multicolumn{2}{|l|}{0.605} & 0.616 & \multicolumn{2}{|c|}{0.610} & \multicolumn{2}{|c|}{0.615} & \multicolumn{2}{|c|}{0.617} \\
\hline SEE & \multicolumn{2}{|l|}{0.542} & 0.535 & \multicolumn{2}{|c|}{0.539} & \multicolumn{2}{|c|}{0.536} & \multicolumn{2}{|c|}{0.534} \\
\hline $\mathrm{F}$ & \multicolumn{2}{|l|}{33.700} & 35.336 & \multicolumn{2}{|c|}{34.442} & 35.0 & & 35. & \\
\hline $\mathrm{R}_{\text {pred }}^{2}$ & 0.6431 & & 0.6485 & 0.6 & & 0.65 & & 0.6 & \\
\hline SEP & 0.656 & & 0.653 & 0.6 & & 0.6 & & 0.6 & \\
\hline $\mathrm{N}_{\mathrm{C}}$ & 1 & & 1 & 1 & & 1 & & 1 & \\
\hline & & & & Field col & tributior & & & & \\
\hline $\mathrm{S}$ & 0.017 & & 0.016 & 0.0 & & - & & 0.0 & \\
\hline $\mathrm{E}$ & 0.076 & & 0.074 & . & & $0.0^{\circ}$ & & 0.0 & \\
\hline $\mathrm{H}$ & 0.047 & & - & 0.0 & & 0.0 & & 0.0 & \\
\hline $\mathrm{D}$ & - & & 0.081 & 0.0 & & $0.0^{\circ}$ & & 0.0 & \\
\hline A & 0.038 & & 0.036 & 0.0 & & 0.0 & & 0.0 & \\
\hline GATS8e & 0.530 & & 0.511 & 0.5 & & 0.4 & & 0.4 & \\
\hline Mor29m & 0.292 & & 0.282 & 0.2 & & $0.2^{2}$ & & 0.2 & \\
\hline
\end{tabular}

Table S4. Summary of QSAR results based on superimposition III.

\begin{tabular}{|c|c|c|c|c|c|c|c|c|c|}
\hline & CoMFA & \multicolumn{10}{|c|}{ CoMSIA } \\
\cline { 2 - 12 } & $\mathrm{SE}$ & $\mathrm{S}$ & $\mathrm{E}$ & $\mathrm{H}$ & $\mathrm{D}$ & $\mathrm{A}$ & $\mathrm{SE}$ & $\mathrm{SH}$ & $\mathrm{SD}$ \\
\hline $\mathrm{R}_{\mathrm{cv}}^{2}$ & 0.464 & 0.493 & 0.454 & 0.488 & 0.479 & 0.466 & 0.461 & 0.495 & 0.486 \\
\hline $\mathrm{R}_{\text {ncv }}$ & 0.663 & 0.703 & 0.641 & 0.630 & 0.654 & 0.625 & 0.650 & 0.638 & 0.663 \\
\hline $\mathrm{SEE}$ & 0.513 & 0.494 & 0.530 & 0.537 & 0.519 & 0.541 & 0.522 & 0.531 & 0.512 \\
\hline $\mathrm{F}$ & 20.631 & 15.756 & 18.713 & 17.912 & 19.881 & 17.497 & 19.527 & 18.521 & 20.701 \\
\hline $\mathrm{R}_{\text {pred }}^{2}$ & 0.7173 & 0.6081 & 0.7236 & 0.7592 & 0.7473 & 0.7511 & 0.7237 & 0.7615 & 0.7478 \\
\hline $\mathrm{SEP}$ & 0.647 & 0.645 & 0.653 & 0.632 & 0.638 & 0.645 & 0.649 & 0.628 & 0.634 \\
\hline $\mathrm{N}_{\mathrm{C}}$ & 2 & 3 & 2 & 2 & 2 & 2 & 2 & 2 & 2 \\
\hline \multicolumn{7}{|c|}{} & \multicolumn{7}{|c|}{ Field contribution } & & & \\
\hline $\mathrm{S}$ & 0.117 & 0.4390 & - & - & - & - & 0.038 & 0.035 & 0.038 \\
\hline $\mathrm{E}$ & 0.178 & - & 0.200 & - & - & - & 0.199 & - & - \\
\hline $\mathrm{H}$ & - & - & - & 0.147 & - & - & - & 0.145 & - \\
\hline
\end{tabular}




\begin{tabular}{|c|c|c|c|c|c|c|c|c|c|}
\hline $\mathrm{D}$ & - & - & - & - & 0.221 & - & - & - & 0.217 \\
\hline $\mathrm{A}$ & - & - & - & - & - & 0.128 & - & - & - \\
\hline GATS8e & 0.583 & 0.390 & 0.632 & 0.647 & 0.635 & 0.653 & 0.609 & 0.626 & 0.611 \\
\hline Mor29m & 0.122 & 0.220 & 0.168 & 0.205 & 0.144 & 0.219 & 0.154 & 0.193 & 0.133 \\
\hline \multicolumn{10}{|c|}{ CoMSIA } \\
\hline & SA & $\mathrm{EH}$ & $\mathrm{ED}$ & EA & $\mathrm{HD}$ & HA & DA & SEH & SED \\
\hline $\mathrm{R}_{\mathrm{cv}}^{2}$ & 0.474 & 0.471 & 0.449 & 0.449 & 0.489 & 0.477 & 0.467 & 0.474 & 0.454 \\
\hline $\mathrm{R}_{\mathrm{ncv}}^{2}$ & 0.633 & 0.671 & 0.693 & 0.666 & 0.681 & 0.651 & 0.678 & 0.678 & 0.700 \\
\hline SEE & 0.535 & 0.506 & 0.490 & 0.510 & 0.499 & 0.522 & 0.501 & 0.501 & 0.484 \\
\hline $\mathrm{F}$ & 18.137 & 21.447 & 23.672 & 20.970 & 22.390 & 19.599 & 22.157 & 22.106 & 24.516 \\
\hline $\mathrm{R}_{\text {pred }}^{2}$ & 0.7541 & 0.7443 & 0.7392 & 0.7264 & 0.7688 & 0.7700 & 0.7681 & 0.7439 & 0.7401 \\
\hline SEP & 0.641 & 0.643 & 0.656 & 0.656 & 0.632 & 0.639 & 0.645 & 0.641 & 0.653 \\
\hline $\mathrm{N}_{\mathrm{C}}$ & 2 & 2 & 2 & 2 & 2 & 2 & 2 & 2 & 2 \\
\hline \multicolumn{10}{|c|}{ Field contribution } \\
\hline $\mathrm{S}$ & 0.035 & - & - & - & - & - & - & 0.033 & 0.033 \\
\hline $\mathrm{E}$ & - & 0.184 & 0.179 & 0.186 & - & - & - & 0.178 & 0.173 \\
\hline $\mathrm{H}$ & - & 0.135 & - & - & 0.132 & 0.132 & - & 0.130 & - \\
\hline $\mathrm{D}$ & - & - & 0.195 & - & 0.197 & - & 0.203 & - & 0.189 \\
\hline $\mathrm{A}$ & 0.129 & - & - & 0.121 & - & 0.117 & 0.122 & - & - \\
\hline GATS8e & 0.634 & 0.537 & 0.522 & 0.542 & 0.542 & 0.568 & 0.546 & 0.519 & 0.503 \\
\hline Mor29m & 0.202 & 0.143 & 0.105 & 0.150 & 0.129 & 0.184 & 0.128 & 0.139 & 0.102 \\
\hline \multicolumn{10}{|c|}{ CoMSIA } \\
\hline & SEA & SHD & SHA & SDA & EHD & EHA & EDA & HDA & SEHD \\
\hline $\mathrm{R}_{\mathrm{cv}}^{2}$ & 0.453 & 0.491 & 0.479 & 0.470 & 0.455 & 0.445 & 0.451 & 0.455 & 0.455 \\
\hline $\mathrm{R}_{\mathrm{ncv}}^{2}$ & 0.674 & 0.687 & 0.658 & 0.686 & 0.640 & 0.631 & 0.641 & 0.638 & 0.641 \\
\hline SEE & 0.504 & 0.494 & 0.517 & 0.495 & 0.518 & 0.524 & 0.517 & 0.519 & 0.517 \\
\hline $\mathrm{F}$ & 21.717 & 23.042 & 20.177 & 22.919 & 39.037 & 37.649 & 39.236 & 38.831 & 39.330 \\
\hline $\mathrm{R}_{\text {pred }}^{2}$ & 0.7253 & 0.7697 & 0.7707 & 0.7686 & 0.6777 & 0.6704 & 0.6761 & 0.6910 & 0.6788 \\
\hline SEP & 0.653 & 0.630 & 0.638 & 0.643 & 0.637 & 0.643 & 0.640 & 0.637 & 0.637 \\
\hline $\mathrm{N}_{\mathrm{C}}$ & 2 & 2 & 2 & 2 & 1 & 1 & 1 & 1 & 1 \\
\hline \multicolumn{10}{|c|}{ Field contribution } \\
\hline$S$ & 0.034 & 0.033 & 0.032 & 0.034 & - & - & - & - & 0.013 \\
\hline $\mathrm{E}$ & 0.182 & - & - & - & 0.076 & 0.079 & 0.077 & - & 0.075 \\
\hline $\mathrm{H}$ & - & 0.127 & 0.128 & - & 0.062 & 0.064 & - & 0.063 & 0.061 \\
\hline $\mathrm{D}$ & - & 0.190 & - & 0.198 & 0.085 & - & 0.086 & 0.087 & 0.084 \\
\hline $\mathrm{A}$ & 0.118 & - & 0.114 & 0.118 & - & 0.052 & 0.051 & 0.051 & - \\
\hline GATS8e & 0.523 & 0.523 & 0.549 & 0.526 & 0.501 & 0.519 & 0.507 & 0.515 & 0.495 \\
\hline Mor29m & 0.143 & 0.126 & 0.176 & 0.123 & 0.276 & 0.286 & 0.280 & 0.294 & 0.273 \\
\hline \multicolumn{10}{|c|}{ CoMSIA } \\
\hline & \multicolumn{2}{|c|}{ SEHA } & SEDA & \multicolumn{2}{|c|}{ SHDA } & \multicolumn{2}{|c|}{ EHDA } & \multicolumn{2}{|c|}{ SEHDA } \\
\hline $\mathrm{R}_{\mathrm{cv}}^{2}$ & \multicolumn{2}{|c|}{0.445} & 0.451 & \multicolumn{2}{|c|}{0.455} & \multicolumn{2}{|c|}{0.455} & \multicolumn{2}{|c|}{0.455} \\
\hline $\mathrm{R}_{\mathrm{ncv}}^{2}$ & \multicolumn{2}{|l|}{0.633} & 0.643 & \multicolumn{2}{|c|}{0.640} & \multicolumn{2}{|c|}{0.649} & \multicolumn{2}{|c|}{0.650} \\
\hline SEE & \multicolumn{2}{|l|}{0.523} & 0.516 & \multicolumn{2}{|c|}{0.518} & \multicolumn{2}{|c|}{0.511} & \multicolumn{2}{|c|}{0.510} \\
\hline
\end{tabular}




\begin{tabular}{|c|c|c|c|c|c|}
\hline $\mathrm{F}$ & 37.937 & 39.539 & 39.117 & 40.660 & 40.923 \\
\hline $\mathrm{R}_{\text {pred }}^{2}$ & 0.6712 & 0.6773 & 0.6916 & 0.6843 & 0.6846 \\
\hline $\mathrm{SEP}$ & 0.643 & 0.640 & 0.637 & 0.637 & 0.637 \\
\hline $\mathrm{N}_{\mathrm{C}}$ & 1 & 1 & 1 & 1 & 1 \\
\hline \multicolumn{7}{|c|}{ Field contribution } \\
\hline $\mathrm{S}$ & 0.013 & 0.013 & 0.013 & - & 0.012 \\
\hline $\mathrm{E}$ & 0.078 & 0.076 & - & 0.072 & 0.072 \\
\hline $\mathrm{H}$ & 0.063 & - & 0.063 & 0.059 & 0.058 \\
\hline $\mathrm{D}$ & - & 0.085 & 0.086 & 0.081 & 0.080 \\
\hline $\mathrm{A}$ & 0.051 & 0.050 & 0.051 & 0.048 & 0.047 \\
\hline GATS8e & 0.512 & 0.501 & 0.508 & 0.477 & 0.471 \\
\hline Mor29m & 0.282 & 0.276 & 0.280 & 0.263 & 0.260 \\
\hline
\end{tabular}

$\mathrm{R}_{\mathrm{cv}}{ }_{\mathrm{c}}=$ cross-validated correlation coefficient using the leave-one-out methods;

$\mathrm{R}^{2}{ }_{\text {ncv }}=$ Non-cross-validated correlation coefficient; $\mathrm{SEE}=$ Standard error of estimate; $\mathrm{F}=$ Ratio of

$\mathrm{R}^{2}{ }_{\text {ncv }}$ explained to unexplained $=\mathrm{R}^{2}{ }_{\text {ncv }} /\left(1-\mathrm{R}^{2}{ }_{\text {ncv }}\right)$;

$\mathrm{R}^{2}$ pred $=$ Predicted correlation coefficient for the test set of compounds; SEP= Standard error of prediction; $\mathrm{N}_{\mathrm{C}}=$ Optimal number of principal components; $\mathrm{S}=$ steric, E=electrostatic, $\mathrm{H}=$ hydrophobic, $\mathrm{D}=$ hydrogen bond donor, $\mathrm{A}=$ hydrogen bond acceptor.

Table S5. Actual and Optimal CoMFA predicted $\mathrm{pK}_{\mathrm{i}}$ of training and test sets.

\begin{tabular}{|c|c|c|c|}
\hline Compound & Observed & Predicted & Residual \\
\hline 1 & 8.6402 & 8.615 & -0.0252 \\
\hline 2 & 5.7794 & 5.781 & 0.0016 \\
\hline 3 & 7.2708 & 7.15 & -0.1208 \\
\hline 4 & 8.1068 & 8.007 & -0.0998 \\
\hline 5 & 7.3990 & 7.406 & 0.007 \\
\hline 6 & 7.4989 & 7.417 & -0.0819 \\
\hline 7 & 7.7696 & 7.476 & -0.2936 \\
\hline 8 & 8.6635 & 8.768 & 0.1045 \\
\hline 9 & 6.6234 & 7.307 & 0.6836 \\
\hline 10 & 8.4318 & 7.83 & -0.6018 \\
\hline 11 & 7.8508 & 7.727 & -0.1238 \\
\hline 12 & 7.3958 & 7.35 & -0.0458 \\
\hline 13 & 8.0862 & 7.752 & -0.3342 \\
\hline 14 & 6.9066 & 7.583 & 0.6764 \\
\hline 15 & 6.3556 & 6.767 & 0.4114 \\
\hline 16 & 6.6517 & 6.67 & 0.0183 \\
\hline 17 & 7.9208 & 7.781 & -0.1398 \\
\hline 18 & 8.2218 & 8.179 & -0.0428 \\
\hline 19 & 7.5229 & 7.278 & -0.2449 \\
\hline 20 & 7.3872 & 7.367 & -0.0202 \\
\hline 21 & 7.3979 & 7.39 & -0.0079 \\
\hline 22 & 7.2716 & 7.285 & 0.0134 \\
\hline & & & \\
\hline & & & \\
\hline
\end{tabular}




\begin{tabular}{|c|c|c|c|}
\hline 23 & 7.0899 & 7.461 & 0.3711 \\
\hline 24 & 7.2396 & 7.553 & 0.3134 \\
\hline 25 & 9.3665 & 9.489 & 0.1225 \\
\hline 26 & 8.0000 & 7.97 & -0.03 \\
\hline 27 & 8.8125 & 8.733 & -0.0795 \\
\hline 28 & 7.6925 & 7.715 & 0.0225 \\
\hline 29 & 8.9245 & 8.96 & 0.0355 \\
\hline 30 & 6.5214 & 6.596 & 0.0746 \\
\hline 31 & 8.5867 & 8.653 & 0.0663 \\
\hline 32 & 7.8210 & 7.765 & -0.056 \\
\hline 33 & 6.6716 & 6.678 & 0.0064 \\
\hline
\end{tabular}

Table S6. Actual and Optimal CoMSIA predicted $\mathrm{pK}_{\mathrm{i}}$ of training and test sets.

\begin{tabular}{|c|c|c|c|}
\hline Compound & Observed & Predicted & Residual \\
\hline 1 & 8.6402 & 8.747 & 0.1068 \\
\hline 2 & 5.7794 & 5.747 & -0.0324 \\
\hline 3 & 7.2708 & 7.116 & -0.1548 \\
\hline 4 & 8.1068 & 7.876 & -0.2308 \\
\hline 5 & 7.3990 & 7.43 & 0.031 \\
\hline 6 & 7.4989 & 7.493 & -0.0059 \\
\hline 7 & 7.7696 & 7.638 & -0.1316 \\
\hline 8 & 8.6635 & 8.98 & 0.3165 \\
\hline 9 & 6.6234 & 7.226 & 0.6026 \\
\hline 10 & 8.4318 & 7.778 & -0.6538 \\
\hline 11 & 7.8508 & 7.574 & -0.2768 \\
\hline 12 & 7.3958 & 7.507 & 0.1112 \\
\hline 13 & 8.0862 & 7.816 & -0.2702 \\
\hline 14 & 6.9066 & 7.48 & 0.5734 \\
\hline 15 & 6.3556 & 6.903 & 0.5474 \\
\hline 16 & 6.6517 & 6.7 & 0.0483 \\
\hline 17 & 7.9208 & 7.822 & -0.0988 \\
\hline 18 & 8.2218 & 8.069 & -0.1528 \\
\hline 19 & 7.5229 & 7.531 & 0.0081 \\
\hline 20 & 7.3872 & 7.561 & 0.1738 \\
\hline 21 & 7.3979 & 7.551 & 0.1531 \\
\hline 22 & 7.2716 & 7.361 & 0.0894 \\
\hline 23 & 7.0899 & 7.142 & 0.0521 \\
\hline 24 & 7.2396 & 7.332 & 0.0924 \\
\hline 25 & 9.3665 & 9.422 & 0.0555 \\
\hline 26 & 8.0000 & 7.929 & -0.071 \\
\hline 27 & 8.8125 & 8.784 & -0.0285 \\
\hline 28 & 7.6925 & 7.64 & -0.0525 \\
\hline 29 & 8.9245 & 8.931 & 0.0065 \\
\hline
\end{tabular}




\begin{tabular}{|l|l|l|l|}
\hline 30 & 6.5214 & 6.536 & 0.0146 \\
\hline 31 & 8.5867 & 8.623 & 0.0363 \\
\hline 32 & 7.8210 & 7.868 & 0.0470 \\
\hline 33 & 6.6716 & 6.678 & 0.0064 \\
\hline
\end{tabular}

Table S7. Descriptors used in model construction.

\begin{tabular}{ccc}
\hline Symbol & Class & Meaning \\
\hline L2 ${ }_{\mathrm{P}}$ & WHIM & 2nd component size directional WHIM index/weighted by \\
& & atomic polarizabilities \\
MATS4P & 2D autocorrelations & Moran autocorrelation-lag 4/weighted by atomic \\
& & polarizabilities \\
GATS8e & 2D autocorrelations & Geary autocorrelation-lag 8/weighted by atomic masses \\
Mor29m & 3D-MoRSE & 3D-MoRSE-signal 29/weighted by atomic masses \\
\hline
\end{tabular}
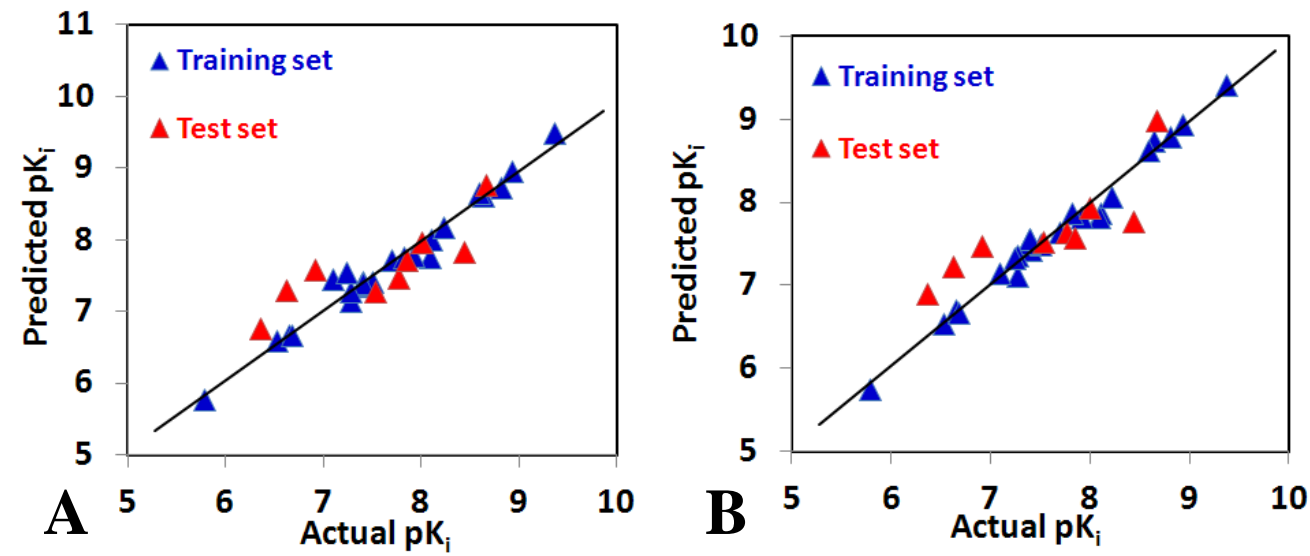

Figure S1. Graphs of the predicted versus the experimental $\mathrm{pK}_{\mathrm{i}}$ values of the optimal models. (A) CoMFA model. (B) CoMSIA model.

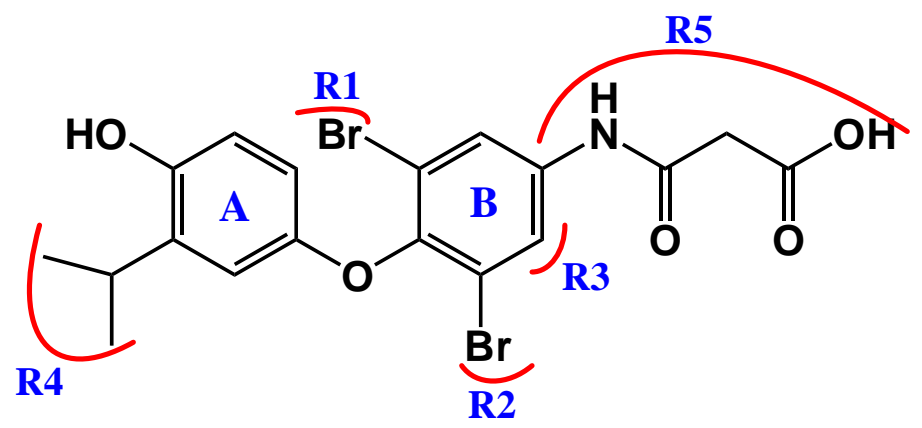

Figure S2. The structure of the most active molecule used in the contour analyses. 


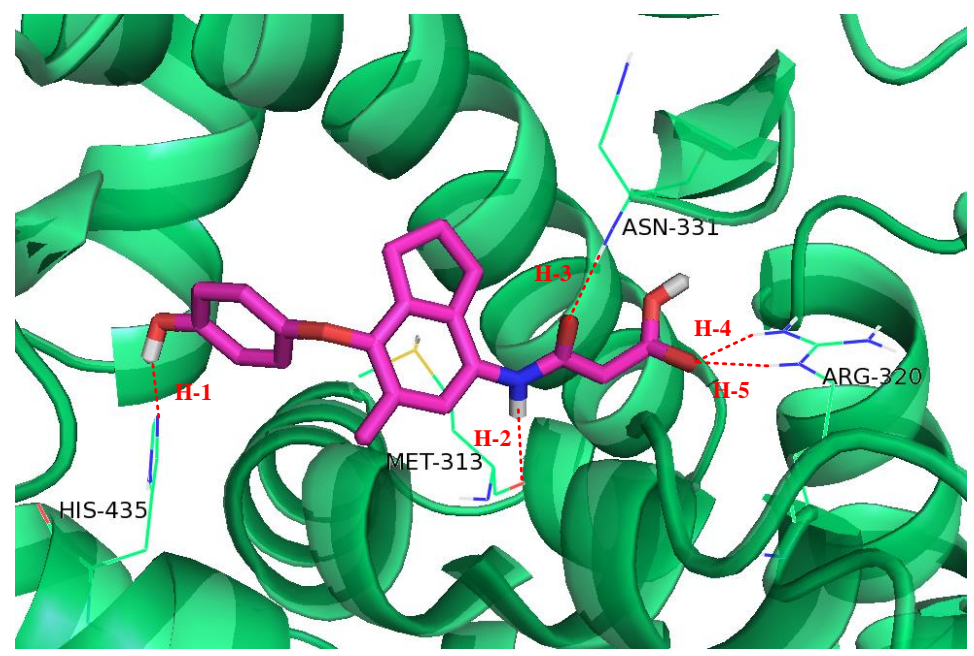

Figure S3. The enlargement for compound 2 in the binding site after molecular docking, which is displayed in stick, H-bonds are shown as dotted black lines, and the nonpolar hydrogens were removed for clarity.

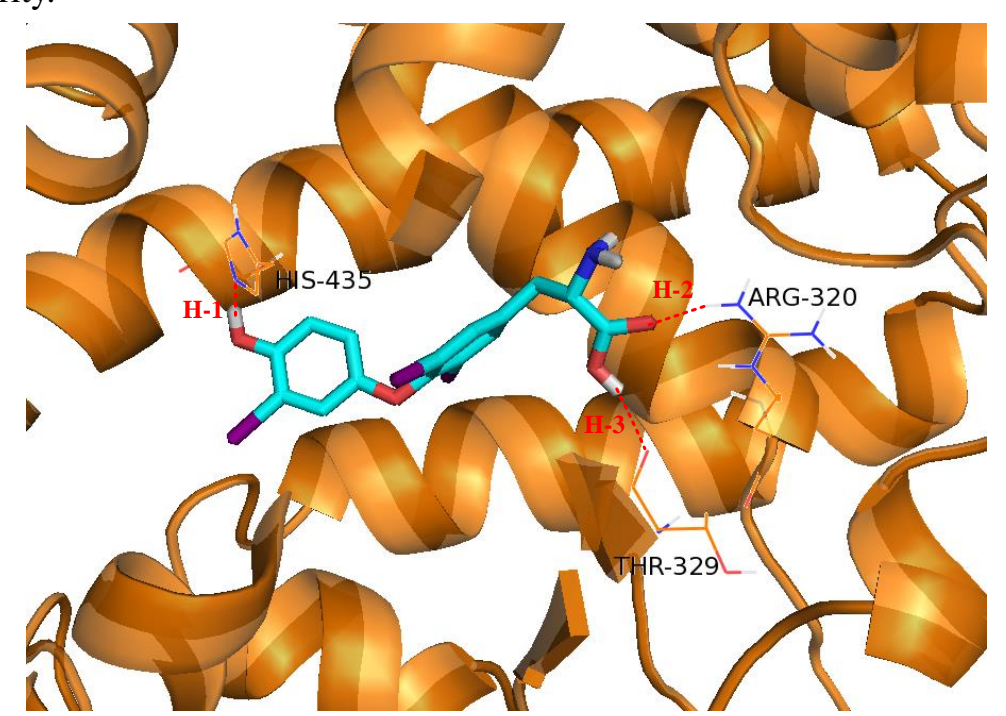

Figure S4. The enlargement for compound 1 in the binding site after molecular docking, which is displayed in stick, H-bonds are shown as dotted black lines, and the nonpolar hydrogens were removed for clarity. 